\title{
How to Write a Research Paper
}

\author{
Andrei V. Alexandrov \\ Stroke Treatment Team, Houston Medical School, University of Texas, Houston, Tex., USA
}

\section{Key Words}

Peer review . Perfect manuscript $\cdot$ Writing skills

\begin{abstract}
Background: Busy strokologists often find little time for scientific writing. They sometimes develop a mental condition equivalent to that known by neurologists as writer's cramp. It may result in permanent damage to academic career. This paper provides advice how to prevent or treat this condition. Methods: Prepare your manuscript following the IMRaD principle (Introduction, Methods, Results, and Discussion), with every part supporting the key message. When writing, be concise. Clearly state your methods here, while data belong to Results. Successful submissions combine quality new data or new thinking with lucid presentation. Results: Provide data that answer the research question. Describe here most important numeric data and statistics, keeping in mind that the shorter you can present them, the better. The scientific community screens abstracts to decide which full text papers to read. Make your point with data, not arguments. Conclusions: Conclusions have to be based on the present study findings. The time of lengthy and unfounded speculations is over. A simple message in a clearly written manuscript will get noticed and may advance our understanding of stroke.
\end{abstract}

Copyright (@) 2004 S. Karger AG, Basel

\section{Introduction}

By now you probably wrote an abstract and submitted it to a stroke conference. Your mentor reminds you several times to start drafting a paper, and you have no idea where to start. As a simple trick, copy and paste your abstract so that Background becomes your introduction. For the rest, follow the IMRaD principle: Introduction, Methods, Results, and Discussion [1-3]. Think what 'take home message' you'd like to deliver and to whom. The title sells the paper.

'Busy strokologists often find little time ... to treat this condition': this introduction concisely describes the study hypothesis, rationale, purpose, and objectives. A threeparagraph introduction is plenty for most topics. Expand with facts from papers previously published by others, among whom you may occasionally find your mentor. Do a thorough literature search for earlier sources dealing with your subject [4-6]. Tell here what is known in the field. You do not need to refer to every paper ever written on this topic. Select key references and remember that for publishing purposes, less is better than more. Consult your mentor as often as possible - he is the senior author after all.

The third paragraph should state the research question [7]. You may take an original paper already published in Cerebrovascular Diseases to use as a template. Formulate the research question clearly since data presentation should provide equally clear answers.

Dr. A.V. Alexandrov

University of Texas

6431 Fannin St, MSB 7.044

Houston, TX 77030 (USA)

Tel.+1 713500 7087, Fax +1 713500 7097, E-Mail avalexandrov@worldnet.att.net 


\section{Subjects and Methods}

The first author drafts the manuscript and determines co-authors [8]. Although general guidelines are available [8], the reality often demands seeking advice from your mentor. Inappropriate inclusion of authors will decrease the likelihood of manuscript acceptance.

Describe subject selection criteria and data collection tools. Make this description detailed enough so that if someone wants to repeat the study, it will be possible. If new imaging technology was used, tell how and by whom these tests were validated. Avoid presenting actual data in this section: 'Study subjects were recruited from 1,215 patients admitted to our stroke unit from August 1999 through August 2002'. Instead say: 'Study subjects were recruited from consecutive patients admitted to our stroke unit. Inclusion criteria were ...'. Methods may disclose power calculations, estimated sample size, and stopping rules.

Provide additional evidence that would increase confidence in the reliability of your methods. Control for biases, validation of research tools, 'blinding' of observers - all of these facts, if established before the study initiation, will strengthen the manuscript. Describe in detail the outcome models or dependent variables. For clinical outcomes or surrogate markers, reference a pivotal trial or study that established their relevance.

Documentation of protection of research subjects is essential. Clearly state if a local ethics committee approved your study. This ensures patients or animal rights protection, particularly if experiments were performed. The author also needs to disclose funding sources and potential for commercial bias such as connections with the pharmaceutical industry. Data safety monitoring, independent data acquisition and analysis during clinical trials and appropriate overseeing committees should be mentioned if applicable.

Major scientific journals currently accept less than $25 \%$ of submitted manuscripts. If rejected, it does not necessarily mean your manuscript is poor. Rejection means that reviewers did not give it a high enough priority. You should not be too disappointed because, after all, you got very good advice how to improve your manuscript. Follow reviewers' suggestions and you increase the likelihood that another esteemed journal will accept it. The most important factors for publication are the quality, novelty, reliability and scientific or clinical importance of your work. A manuscript should disclose new information or a new way of thinking about old information. If not, it will not be published - regardless of how well it is written. Avoid redundant or duplicate publications since these should not be published. Scientific publishing is extremely competitive, and chances are that by the time you conceived the project, 10 other groups were already doing it and 5 others have already published it. Stay on top of current literature and know the limitations of research done by others.

The last paragraph of this section should describe tools of statistical analysis appropriate to study design. Consult a statistician before embarking on a project, work with a statistician to analyze and interpret the data, and have a statistician reviewing the whole manuscript for clarity of statistical analysis and data presentation.

\section{Results}

Your results are the most important part of the manuscript. Present them clearly by avoiding long and confusing sentences. The shorter you can present your data in tables and figures, the better. Remain focused and disciplined. The flurry of numbers and ' $p$ ' values should follow simple logics. Start by describing your study subjects, use actual numbers for study demographics. Avoid opening sentences like: 'Table 1 summarizes our findings in subgroup C'. This makes reviewers frustrated since they have to flip back and forth through pages to understand what was done to study subjects.

Make data presentation so clear and simple that a tired person riding late on an airplane can take your manuscript and get the message at first reading. Very few people can write a perfect manuscript on the first draft. Return to the draft, read it, change cumbersome parts, read other papers and change the draft again, and again, and again. I still do it before I give the manuscript to my co-authors. But do not hold it for too long. Remember, ' 10 other groups ....

Present results to colleagues since they would likely ask for more data or analyses. Most likely the reviewers of any esteemed journal would do the same, so include data in the first draft of your manuscript. The internal review is helpful to determine sufficient data to answer the research question.

Most importantly, provide data relevant to the research question. Observations beyond the primary research question can be included in the manuscript, if they strengthen your case. Remember to stay in focus. If you get lost from the aim of the study, so will be reviewers. Prestigious journals have a strict word limit for papers they accept. You need all this space to deliver the key mes-

\footnotetext{
$\overline{136} \quad \overline{\text { Cerebrovasc Dis 2004;18:135-138 }}$
} 
sage, so do not mess around but concentrate on the essential. Packing manuscript with data is better than splitting the paper into separate small ones.

Mention a statistical test that generated specific ' $p$ ' values or coefficients. Show absolute numbers as well as percentages so that reviewers can judge the significance of your observations. Remember that statistical difference does not necessarily translate into clinical significance.

Make your point with data, not arguments.

\section{Discussion}

This section should start with: 'Our study showed ...' to lucidly summarize your study findings. Discussion is often the weakest part of the manuscript. Do not repeat the introduction. Do not present any new data that were not shown in the results section and avoid repeating data presentation. There is no reason to underline how terrific your results are - let them speak for themselves.

The second paragraph may describe the novelty of your findings or if they parallel previous research. Remember, only the beginners try to refer to all published papers in the field. No esteemed journal can afford the space needed for this. A skillful selection of the most pertinent references demonstrates a command of the relevant literature. Confirmatory research makes passing the review process more difficult. Arbitrarily, the ratio of abstracts to original papers in curriculum vitae should be less than 3 to 1 . If there are too many abstracts, you either have writer's cramp or the quality of your research is insufficient for publication.

The third paragraph may describe how your study contradicts previous research or established dogmas. If there was disagreement about study interpretation by co-authors, mention different conclusions drawn from your results or other studies [9, 10]. Avoid general statements that are not founded in data. Do not provide your opinion how to solve a problem that was not directly evaluated in your study. Do not write a review of all possible mechanisms that you have not accounted for in your study. You can write a short but to-the-point Discussion.

The fourth paragraph should describe study limitations. If you do not discuss study weaknesses, the reviewers will. Study limitations may be contrasted with study strengths. This part may also mention unresolved questions and direction of future research.

The concluding paragraph can summarize the potential significance of your findings and what changes to research or clinical practice your data may support. This is a critical part since it is easy to overestimate the significance of your research. Avoid broad claims and strong statements. Remember that even pioneer break-through studies require independent confirmation. Publication in a peer-reviewed journal means completion of your project and dissemination of research results $[11,12]$.

Clinicians need to develop skills in scientific writing. If you make a significant observation, a proper and fast scientific communication is required [12]. Improving your scientific writing is a life-long process. If and when your papers are rejected, remember that most manuscripts face the same fate. Avoid choosing an inappropriate journal for your manuscript submission. Common reasons for rejection include inappropriate or incomplete statistics; over-interpretation of results; inappropriate or sub-optimal instrumentation; a sample too small or biased; difficult-to-follow writing; insufficient problem statement; inaccuracy or inconsistency of the data reported; incomplete, inaccurate, or outdated review of the literature; insufficient data presented, and defective tables or figures [13-15]. When reading criticism, learn from your mistakes or the advice given to you. While wrestling with reviewers, you will become a better scientific writer but also a better, more critical scientist. In the long run this will make a major difference to your academic career, and probably will also improve your patient care. Most likely, your way of writing will become more evidence based.

An anonymous and probably frustrated academician once said: 'Publish or perish!'. This brutally honest statement should motivate you to learn yet another set of useful skills. Good luck!

\section{Acknowledgment}

The author is not a native English speaker. I am indebted to John Norris, MD, FRCP, for - among many things during fellowship training - his patience with my 'a's and 'the's, and the first lessons in study design, analysis, and presentation. The infamous 'Norris Rules' that he taught his fellows are partly reflected in this paper. 


\section{References}

1 Pakes GE: Writing manuscripts describing clinical trials: A guide for pharmacotherapeutic researchers. Ann Pharmacother 2001;35:770779.

-2 Pamir MN: How to write an experimental research paper. Acta Neurochir Suppl 2002;83: 109-113.

$\checkmark 3$ Kern MJ, Bonneau HN: Approach to manuscript preparation and submission: How to get your paper accepted. Catheter Cardiovasc Interv 2003;58:391-396.

$\checkmark 4$ Cummins RO: Learning to write: Can books help? J Med Educ 1981;56:128-132.

5 Linney BJ: The three R's of writing: Reading, 'riting,' and risking. Physician Exec 1997;23: 59-61.
6 Cupples SA: Publishing the research report. Alzheimer Dis Assoc Disord 1999;13(suppl 1): S123-S130.

7 Wojner AW: Outcomes Management: Applications to Clinical Practice. St Louis, Mosby 2001, pp 69-88.

8 Anonymous: Policy on papers' contributors. Nature 1999;399:393.

Horton R: The hidden research paper. JAMA 2002;287:2775-2778.

10 Clarke M, Alderson P, Chalmers I: Discussion sections in reports of controlled trials published in general medical journals. JAMA 2002;287: 2799-2801.

11 Bossuyt PM, Reitsma JB, Bruns DE, Gatsonis CA, Glasziou PP, Irwig LM, Lijmer JG, Moher D, Rennie D, De Vet HC; STARD Group: Towards complete and accurate reporting of studies of diagnostic accuracy: The STARD initiative. AJR Am J Roentgenol 2003;181:5155.
2 Pearn J: Publication: An ethical imperative. BMJ 1995;310:1313-1315.

13 Bordage G: Reasons reviewers reject and accept manuscripts: The strengths and weaknesses in medical education reports. Acad Med 2001;76:889-896.

14 Samet JM: Dear author - advice from a retiring editor. Am J Epidemiol 1999; 150:433-436.

5 DeBehnke DJ, Kline JA, Shih RD: Research fundamentals: Choosing an appropriate journal, manuscript preparation, and interactions with editors. Research Committee of the Society for Academic Emergency Medicine. Acad Emerg Med 2001;8:844-850. 